\title{
Pre- and postnatal smoking intervention in managed care settings
}

\author{
Michael Wall
}

Maternal smoking has become the most preventable cause of fetal loss and preterm delivery in the United States. In addition, maternal smoking has been associated with postnatal morbidity and mortality from respiratory infections and sudden infant death syndrome. ${ }^{1}$ Approximately $30 \%$ of women who are pregnant and smoke quit at some point in their pregnancy, but postpartum relapse rates in the ensuing $6-12$ months average $60-80 \%{ }^{2}$ In our studies as well as others, the major predictors of continued smoking during pregnancy are daily consumption of cigarettes, low socioeconomic status, and the presence of other smokers in the home. ${ }^{3}$ Similarly, the major predictors of postnatal relapse are socioeconomic status and other smokers in the home. $^{3}$

Managed care capitated organisations have a financial self interest in maternal smoking intervention. There is no other clinical circumstance in which the positive effects of smoking cessation are as immediate as during pregnancy. It has been estimated that every dollar spent in maternal smoking cessation leads to immediate savings of $\$ 3$ and long term savings of about $\$ 7 .{ }^{4}$ One could fund the entire smoking cessation program of an average sized managed care organisation (MCO) for the short and long term health costs of one extremely premature infant.

Effective maternal smoking cessation and relapse prevention in an MCO requires the assemblage of an interactive team including obstetrical providers and their staff, system case managers, maternity service staff, and postnatal providers. Putting together such a team approach in the combination of provider/ individual practice association/hospital(s) health system/insurer can be a formidable task. Our current work has suggested a format that may work for other systems.

We believe that a brief smoking history should be taken at the first prenatal visit. The history should identify current smokers as well as recent quitters. To screen for smoking status we use the following two questions: did you smoke in the month before learning that you are pregnant; and do you smoke now? Further information can then be obtained as indicated by the responses to the two questions. Obstetrical providers should provide brief, office based interventions using the now standard " 4 A's" approach. ${ }^{5}$ Smoking during pregnancy offers at least three different areas for discussion with the mother: her own health, the health of the child, and role modelling ("Most women who smoke do not want their own children to become smokers. How do you feel about that?"). We believe that system based prenatal case management is an essential adjunct to office based intervention. In our current work we are taking a systems "continuous quality improvement" approach to ensure that the smoking history obtained at the provider office is transmitted as soon as possible (1-2 weeks) to system case managers. The case managers then begin their own smoking assessment and intervention. The case management is not limited to smoking as a sole risk factor. Our data show that maternal smoking is strongly associated with other risks such as alcohol, drugs, sexually transmitted diseases, abuse, and depression.

We are currently in the process of linking the providers and case managers to existing hospital based smoking intervention to provide a brief "booster" at the time of delivery. Previous work has shown that we can link postnatal providers via the case managers or hospital based programs. The major challenge for the future is to develop programs directed at other smokers in the home.

1 US Department of Health and Human Services. The health benefits of smoking cessation. A report of the Surgeon General, 1990. Rockville, Maryland: Public Health Service, Centers for Disease Control, Office on Smoking and Health, 1990. (DHHS Publication No (CDC) 90-8416.)

2 Ockene JK. Smoking among women across the life span: prevalence, interventions, and implications for cessation prevalence, interventions, and implications
research. Ann Behav Med 1993;15:135-48.

3 Severson $\mathrm{HH}$, Andrews JA, Lichtenstein E, et al. Predictors of smoking during and after pregnancy: a survey of mothers of newborns. Prev Med 1995; 24:23-8.

4 Marks JS, Koplan JP, Hogue CJR, et al. A cost-benefit/costeffectiveness analysis of smoking cessation for pregnant women. Am f Prev Med 1990; 6:282-9.

5 Smoking Cessation Clinical Practice Guideline Panel. The Agency for Health Care Policy and Research smoking cessation clinical practice guideline. FAMA 1996;275:127080 . 